[3] D. Mukherji et al, Kovove Mater. 53, 2015, 287

Keywords: High-temperature materials, Co-Re alloys, Neutron scattering

\section{MS42-O4 Neutron Total Scattering of Crystalline Materials in the Gigapascal Regime}

Craig L. Bull ${ }^{1}$, Helen Y. Playford ${ }^{1}$, Matthew G. Tucker ${ }^{1}$

1. ISIS Facility, STFC, Rutherford Appleton Lab

email: craig.bull@stfc.ac.uk

Neutron total scattering of disordered-crystalline materials provides direct experimental access to the local (short-range) structure. The ways in which this local structure agrees (or disagrees) with the average (long-range) crystal structure can provide important insight into structure-property relationships. High-pressure neutron diffraction using the Paris-Edinburgh (P-E) pressure cell allows experimenters to explore the ways in which materials are affected by pressure, can reveal new synthetic routes to novel functional materials and has important applications in many areas, including geology, engineering and planetary science. The combination of these two experimental techniques poses unique challenges for both data collection and analysis. In this paper it is shown that, with only minor modifications to the standard P-E press setup, high-quality total scattering data can be obtained from crystalline materials in the gigapascal pressure regime on the PEARL diffractometer at ISIS. The quality of the data is assessed through the calculation of coordination numbers and the use of reverse Monte Carlo (RMC) refinements. The time required to collect data of sufficient quality for detailed analysis is assessed and is found to be of the order of 8 hours for a quartz sample. Finally, data from the perovskite $\mathrm{LaCo0}: 35 \mathrm{Mn} 0: 65 \mathrm{O} 3$ is presented and reveals that PEARL total scattering data offers the potential for the extraction of local structural information from complex materials at high pressure.

Keywords: neutron, high-pressure, total scattering 